\title{
AMT detection research of gas hydrate prospecting in the Qiangtang Basin, Northern Tibet
}

\author{
Dawei Yao, Shumin Wang, Dayong Wang, Yongbo Li, Zhenyu Zhang, Gang Wang, Wei Zhu \\ Institute of Geophysical and Geochemical Exploration, Chinese Academy Geological Science, \\ Langfang, Hebei, People's Republic of China \\ E-mail: yaodawei@igge.cn
}

\begin{abstract}
Qiangtang basin is the largest oil and gas basins of the Tibet plateau, the permafrost distributed widely, having good formed condition and prospecting of gas hydrate. There is carried out audio magnetotelluric (AMT) exploration research in YaHu area in order to find out the basic accumulation conditions of geology and geophysics of the natural gas hydrate in Qiangtang basin. The detection results show that AMT detection had high sensitivity to the permafrost, it could distinguish effectively bottom boundary of permafrost caprock distribution and delineate clearly natural gas migration pathway reservoir distribution. The result of 2-D inversion of geoelectric section showed the thickness of Yahu frozen permafrost is between 50 to 100 meters, the development degree of frozen soil is affected mainly by tectonic fracture zone, sedimentary loose bed, surface water and the factors such as elevation height control. The results further supported that Yahu regions have good conditions of frozen soil cover layer, good rich gas migration channel and reservoir structure.It is a good block which is suitable for accumulation and reserves for gas hydrates.
\end{abstract}

Keywords - the Qiangtang basin; permafrost; natural gas hydrate; $A M T$

\section{INTRODUCTION}

Natural gas hydrate is a solid state material classes. It is formed with relatively low molecular mass of gas (such as methane, ethane, propane, etc.) and water in a certain temperature and pressure conditions. It mainly occurs in the extreme permafrost horizon, epicontinental sea and some sediments at the bottom of the deep water lake. Natural gas hydrate was seen as a potential new energy in the new century as it had high energy density, wide distribution, large scale, buried depth shallow ${ }^{[1]}$. Real samples of natural gas hydrate had successful drilled in the Qilian mountain regions, Tibetan plateau in 2008-2009. It was the first time in the world to find region, and achieved a major breakthrough in the detection research of gas hydrate prospecting ${ }^{[2]}$. Compared with the Qilian mountain regions, Tibet Qiangtang basin has better temperature of natural gas hydrate formation pressure and hydrocarbon source conditions, Because it has lower annual mean ground temperature, relatively thick permafrost and low geothermal gradient and good conditions of hydrocarbon gas ${ }^{[3]}$. Carrying out the geophysical exploration of gas hydrate in the Qiangtang basin has important research significance in prospecting breakthrough and the perfect frozen natural gas hydrate reservoiring theory ${ }^{[4]}$. The author had taken part in the audio magnetotelluric (AMT) detection in Yahu in 2014-2015, surveied permafrost distribution, fracture structure formed stratigraphic distribution, the basic accumulation conditions of geology and geophysics of the natural gas hydrate in the area,which provide the basis for gas hydrates exploration and resource of permafrost in Yahu area in Qiangtang basin.

\section{METHOD}

There were three detection lines set in the Yahu area of Qiangtang basin block. (Fig.1.) V5-2000 magnetotelluric sounding device and the way of tensor were used in the field measurement, 5 components data was collected respectively (Ex, Ey, Hx, Hy, Hz.x, y for the horizontal direction, and $\mathrm{z}$ for the vertical direction). The observation frequency range is 1$10400 \mathrm{HZ}$, acquising for 60 minutes at a time, MN polar distance of 50 meters. Station of the cloth extremely way mainly adopts '+' word, sometimes also used the ' $T$ ' type or ' $\mathrm{L}$ ' type. Y6, Y9 and Y10 section length is $10 \mathrm{~km}$, distance of $100 \mathrm{~m}$. Instrument consistency test and measuring point repeat checking the data quality are used to control date quality.
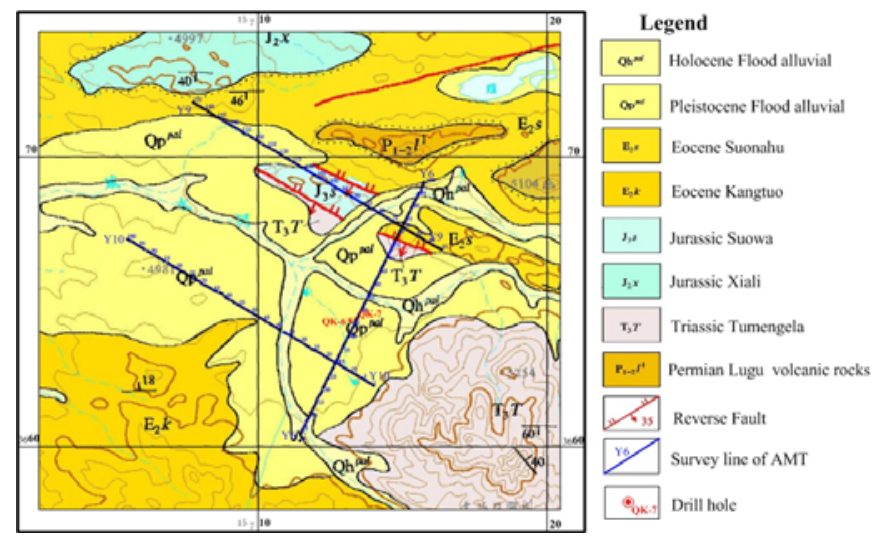

Fig. 1. The line layout and geologic structure

The electromagnetic signal spectrum from $5 \mathrm{kHZ}$ to $800 \mathrm{HZ}$ and 5 to $0.05 \mathrm{HZ}$ frequency band were called "dead band". It was relatively difficult to obtain high quality data in "dead band" because of the low signal noise ratio (SNR) cause by the existence of minimum and the extremely weak signal amplitude. The permafrost buried depth of the corresponding frequency points fall within the dead band, it is critical to deal with the dead band data. This article mainly adopted artificial sleeking, "Rhoplus" analysising, and based on the layered media resistivity and phase of each numerical technology and other advanced processing method to handle "dead band" data. 
Good results were obtained, the work laid a solid foundation

for subsequent data analysising and data inversion. (Fig.2., 3.)
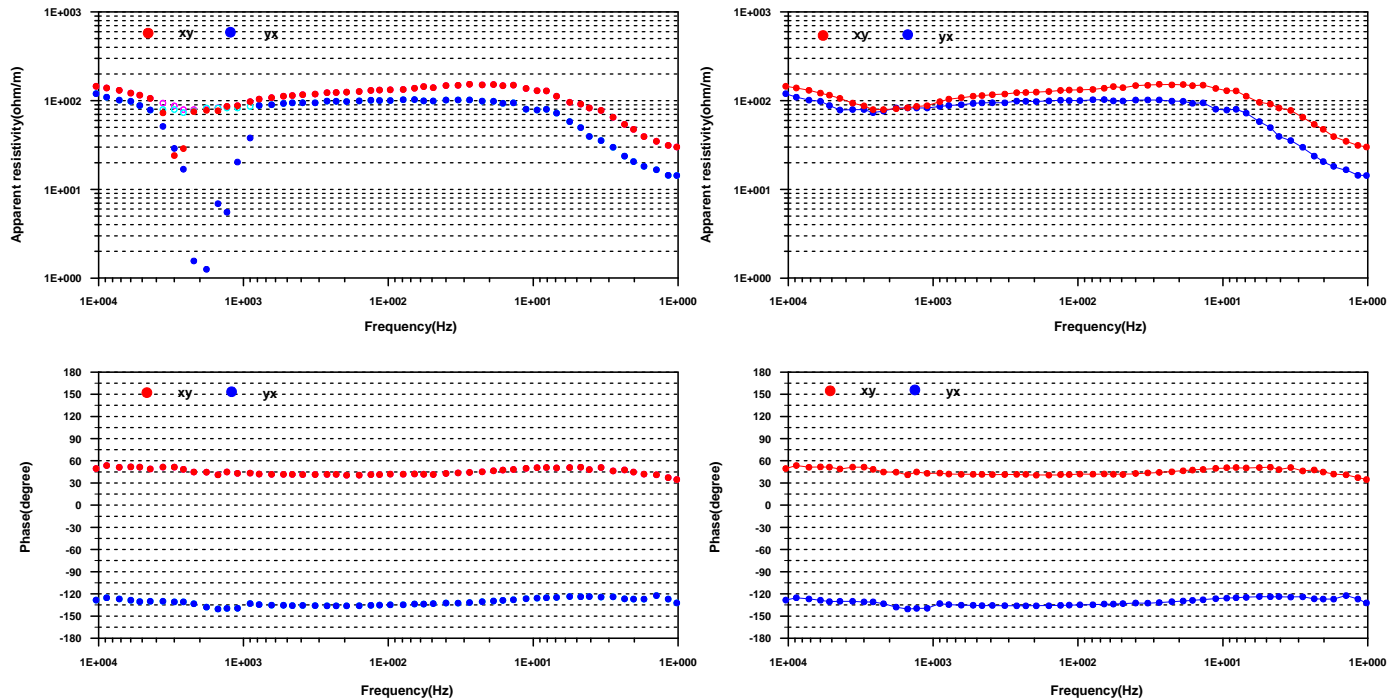

(a) Y6-41 point mapping of the original deep curve

(b) Y6-41 points after editing the results

Fig. 2. Processing results of Y6-41 point measurement points based on layered medium resistivity and phase interaction technique
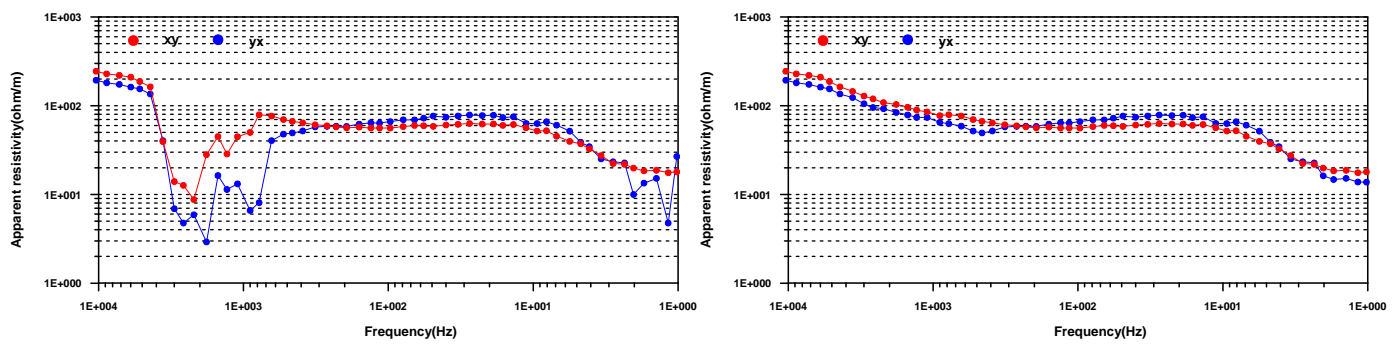

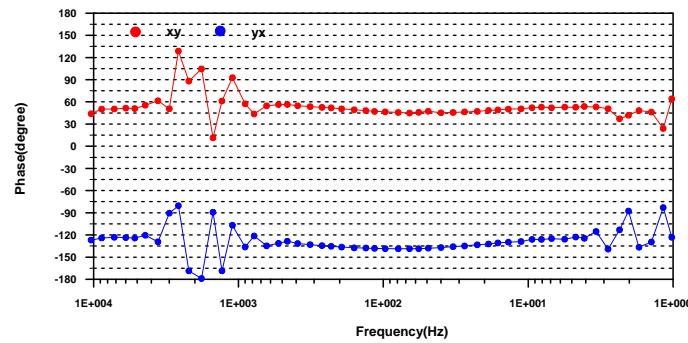

(a) Y6-46 point mapping of the original deep curve

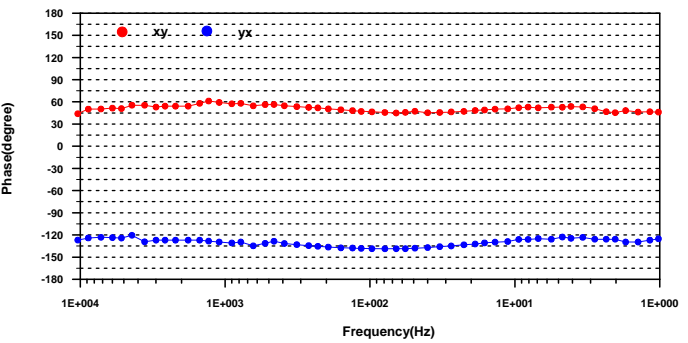

(b) Y6-46 points after editing the results

Fig. 3. Rhoplus analysis of the results of the Y6-46 measurement points

\section{ANOMALY INTERPRETATION}

This paper use the nonlinear conjugate gradient (NLCG) 2d automatic inversion technique, which would solve largescale matrix, preconditions and converted to solve large-scale matrix and vector product, and does not need to be calculated each iteration the large matrix, so effectively reduce the computation time and computer memory footprint. It implemented the search step nonlinear, and improved the degree of nonlinear NLCG, mading NLCG method inversion quickly and result realiable in the process of inversion. NLCG inversion method was more frequently used in processing magnetotelluric data, and the actual effect was good. Finally, there was the calculation of inversion profile within the scope of the underground 2-d resistivity profile (Fig. 4.).
Overall, three profils surface relief is relatively flat. Twodimensional resistivity profile showed that from surface to underground $1000 \mathrm{~m}$ range internal resistance rate could be divided into three layers, characterized by high - medium high - low distribution. Shallow was a high resistivity layer thickness range within 0 - $150 \mathrm{~m}$, its relatively continuous forms in Y6 and Y10 line. Below was a layer of medium high resistivity layer, which distributed within a wide range, both ends gradually annihilated in Y6 line, and appeared only in small points, to large fault disappear in Y9 and Y10 line. The bottom is the low resistance layer, which displayed only a very thin layer in Y6 line and expanded with the lower resistivity in Y9 and Y10 line 


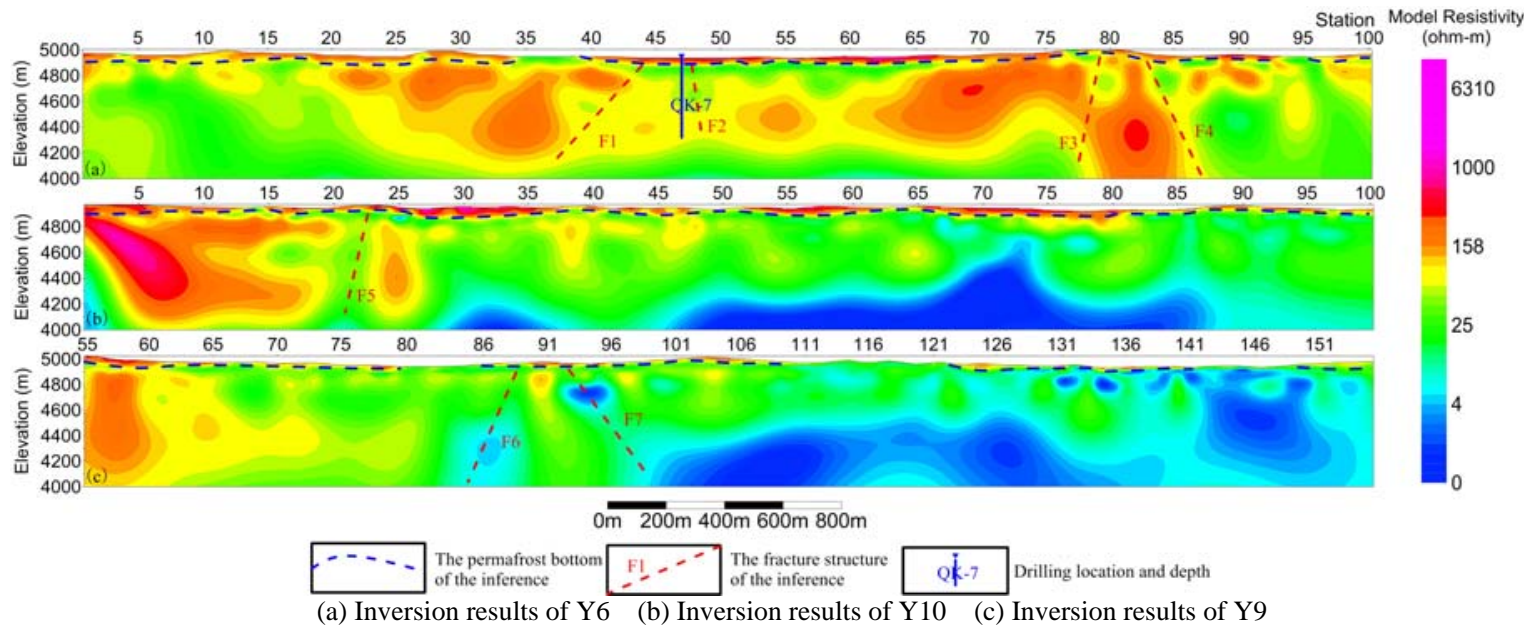

Fig. 4. $2 \mathrm{~d}$ inversion result with terrain
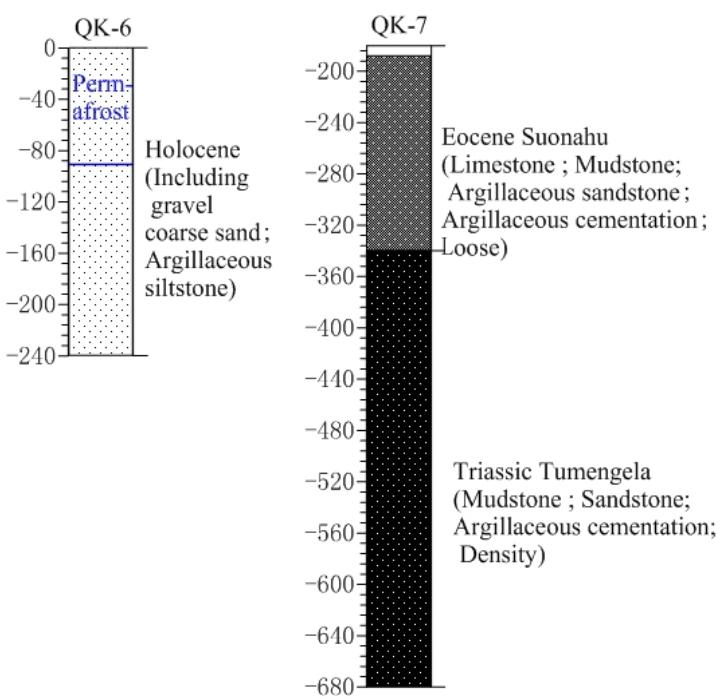

Fig. 5. Drilling result diagram

There was a south west pour fault F1 and an approximate vertical fault F2 near 45 point, and a fault zone which had a south west pour fault F3 and a north east pour fault F4 near 80 point in Y6 line. There was a south pour fault F5 near 45 point in Y10 line. There was a fault zone near 90 point in Y9 line which had a south pour fault F6 and a north pour fault F7. The specific location was shown in the Fig. 4. The fault F3 exposed to the surface of the fracture structure on the geological map annotation. The inference results are basically consistent with geological data, which was according to the distribution characteristics of resistivity.

In combination with regional geology and drilling data and characteristics of resistivity, we thought that in Fig.4 shallow high resistivity anomaly reflection of thin layer was permafrost. It was draw out the tundra line on three sections, which showed the thickness of the permafrost in $50-150 \mathrm{~m}$ range. The permafrost development was poor, and the bottom was discontinuity in Y9 line. Low resistance interlining was a reflection of the Eocene Suonahu between two high resistance, a reflection of its high resistance layer of the Triassic Tumengela in Y6 line. High resistance that occurs in the small point anomalies was the Triassic Tumengala reflected, medium high resistance that occurs in the large point anomalies was the Eocene Suonahu reflected. The inversion results consist

with the results of drilling: (1) The inversion results of permafrost floor was around $80 \mathrm{~m}$ that was consistent with drilling results; (2) $315 \mathrm{~m}$ was the dividing line between the Eocene Suonahu and the Triassic Tumengala in the diagram drilling, and the inversion results are also here for the electrical interface.

\section{CONCLUSIONS}

It was effective and feasible to the morphology of fracture structure and stratigraphic distribution by AMT on the 
permafrost distribution situation in Yahu regions of natural gas hydrate prospective area Qiangtang basin Tibet.

(1) The AMT was an effective method to delineate the natural gas hydrate cover layer of permafrost because of the shallow high resolving power, reflecting the clear of permafrost.

(2) The AMT could be delineated natural gas migration pathway clearly and reservoir fracture structure.

(3) Permafrost thickness generally was 50 - $100 \mathrm{~m}$ in Yahu regions. The degree of permafrost development have a lot to do with with the structure, surface water , fracture zone, sedimentary loose bed elevation height and other factors. Yahu regions was the accumulation of gas hydrates and the favorable reservoir blocks because it have good cover layer of permafrost, developed gas migration channel and reservoir structure.

\section{REFERENCES}

[1] MAKOGON Y F, HOLDITCH S A, MAKOGON T Y. Natural gas hydrates-A potential energy source for the 21st Century[J] Journal of Petroleum Science and Engineering, 2007, 56(1-3):14-31.

[2] ZHU Youhai, ZHANG Yongqin, WEN Huaijun, etc. 2009, Gas Hydrates in the Qilian Mountain Permafrost, Qinghai, Northwest China [J] Acta Geologica Sinica:83(11):1761-1770.

[3] ZHU Youhai, Lu Zhen-quan, Xie Xilin. 2011, Qinghai-Tibet plateau potential distribution area of natural gas hydrate prediction [J]. Geological bulletin of China: 30 (12) : 1918-1926.

[4] Wang Ping-kang, ZHU Youhai, Zhang Xu-hui, etc. 2015, Permafrost in Qiangtang basin structure characteristics and its impact on natural gas hydrate reservoir [J]. Sedimentary Geology and Tethyan Geology: 01:57-67. 